\title{
Independence of Roseman moves including triple points
}

\author{
KENGO KAWAMURA \\ KANAKO OSHIRO \\ KOKORO TANAKA
}

\begin{abstract}
The Roseman moves are seven types of local modifications for surface-link diagrams in 3-space which generate ambient isotopies of surface-links in 4-space. In this paper, we focus on Roseman moves involving triple points, one of which is the famous tetrahedral move, and discuss their independence. For each diagram of any surface-link, we construct a new diagram of the same surface-link such that any sequence of Roseman moves between them must contain moves involving triple points (and the number of triple points of the two diagrams are the same). Moreover, we find a pair of diagrams of an $S^{2}$-knot such that any sequence of Roseman moves between them must involve at least one tetrahedral move.
\end{abstract}

57Q45; 57R45

\section{Introduction}

Throughout this paper, we work in the smooth category. A surface-link (or a $\Sigma^{2}-$ link) is a submanifold of 4 -space $\mathbb{R}^{4}$ diffeomorphic to a closed surface $\Sigma^{2}$. If it is connected, then it is called a surface-knot (or a $\Sigma^{2}-k n o t$ ). Surface-links are not necessarily assumed to be orientable in this paper. Two surface-links are said to be equivalent if they can be deformed into each other through an isotopy of $\mathbb{R}^{4}$.

A diagram of a surface-link is its image via a generic projection from $\mathbb{R}^{4}$ to $\mathbb{R}^{3}$, equipped with the height information as follows: in a neighborhood of each double point, there are intersecting 2-disks such that one is higher than the other with respect to the $4^{\text {th }}$ coordinate that was dropped by the projection. The height information is indicated by removing the regular neighborhood of the double point in the lower disk along the double point curves. A diagram is regarded as a disjoint union of connected compact orientable surfaces, each of which is called a sheet, and is composed of the four kinds of local pictures shown in Figure 1, each of which is the image of a neighborhood of a typical point: a regular point, a double point, an isolated triple point or an isolated branch point. 

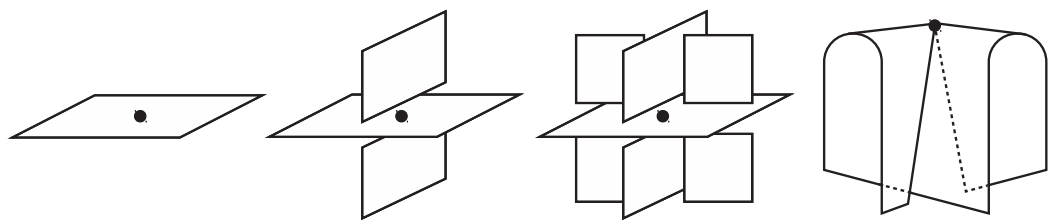

Figure 1: Local pictures of the projection image. From left to right: a regular point, double point, triple point, and branch point.

Two surface-link diagrams are said to be equivalent if they are related by (ambient isotopies of $\mathbb{R}^{3}$ and) a finite sequence of the seven types of Roseman moves, shown in Figure 2, where we omit height information for simplicity, and the symbols B, T and D stand for branch point, triple point and double point curve, respectively. Roseman [17] proved that two surface-links are equivalent if and only if they have equivalent diagrams. We refer to Carter, Kamada and Saito [4], and Carter and Saito [3] for more details on surface-links and their diagrams.

In this paper, we focus on Roseman moves involving triple points, that is, the three moves of type T1, T2 and BT, and discuss their independence. The move of type T2 is also called the tetrahedral move and is closely related to the Zamolodchikov equation, which is a higher dimensional analogue of the Yang-Baxter equation; see [3, Chapter 6] for details.
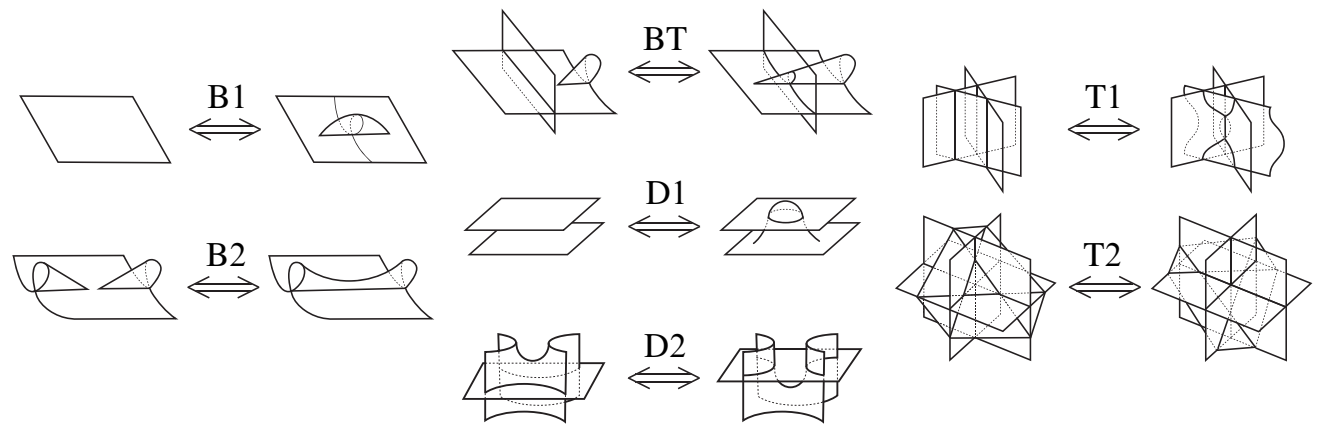

\section{A Problems}

Independence of Roseman moves is already well understood in terms of local moves. We summarize the known results below. The first and third were proved by Kawamura [12] and the second was proved by Homma and Nagase [7] and Yashiro [23]. 
- Type B1 can be realized by a finite sequence of types B2 and D1.

- Type B2 can be realized by a finite sequence of types B1 and D2.

- No types other than B1 and B2 can be realized by the other six types.

However, these results do not give us an answer to the following question: for two diagrams of a surface-link, what types should appear in a sequence of Roseman moves between them? For example, consider the following problem.

Problem 1.1 (1) Are there two diagrams of a surface-link such that any sequence of Roseman moves between them must contain moves involving branch points?

(2) Are there two diagrams of a surface-link such that any sequence of Roseman moves between them must contain moves involving triple points?

Many studies of Problem 1.1(1) have been made; see, for example, Oshiro and Tanaka [16], Satoh [19] and Takase and Tanaka [21]. On the contrary, there are only a few results on Problem 1.1(2), Jabłonowski [9], for example. To make the problem concrete, we define the notion of the $S$-dependence of diagrams for a subset $S$ of the set consisting of the seven types of Roseman moves, and formulate our problem.

Definition 1.2 For a subset $S$ of the set $\{\mathrm{B} 1, \mathrm{~B} 2, \mathrm{D} 1, \mathrm{D} 2, \mathrm{~T} 1, \mathrm{~T} 2, \mathrm{BT}\}$ consisting of the seven types of Roseman moves, two diagrams of a surface-link are said to be $S$-dependent if any sequence of Roseman moves between them contains at least one move in $S$.

Problem 1.3 For a subset $S$ of the set $\{\mathrm{B} 1, \mathrm{~B} 2, \mathrm{D} 1, \mathrm{D} 2, \mathrm{~T} 1, \mathrm{~T} 2, \mathrm{BT}\}$, are there two diagrams of a surface-link such that they are $S$-dependent? We note that if we choose $S$ as $\{\mathrm{B} 1, \mathrm{~B} 2, \mathrm{BT}\}$ (resp. $\{\mathrm{T} 1, \mathrm{~T} 2, \mathrm{BT}\}$ ) then the problem is equivalent to Problem 1.1(1) (resp. (2)). We also note that if $S^{\prime} \subset S$ then an $S^{\prime}$-dependent pair is $S$-dependent by definition. Since we are now interested in Roseman moves involving triple points, we will take $S$ as a subset of $\{\mathrm{T} 1, \mathrm{~T} 2, \mathrm{BT}\}$ in what follows.

\section{$1 B$ Results}

Jabłonowski [9] observed a $\{\mathrm{T} 1, \mathrm{~T} 2, \mathrm{BT}\}$-dependence of surface-link diagrams, and showed that there is a pair of diagrams of the trivial $\left(S^{2} \cup T^{2}\right)$-link such that the pair is $\{\mathrm{T} 1, \mathrm{~T} 2, \mathrm{BT}\}$-dependent and each of the two diagrams has no triple points. Surface-link diagrams which he constructed are oriented, and have multicomponents and positive genus, which were crucial conditions in his proof. In Section 2, we will generalize his result for any surface-link (including unoriented surface-links, surface-knots and $S^{2}$-links), and prove the following. 
Theorem 1.4 For each diagram $D$ of any surface-link $F$, there is a diagram $D^{\prime}$ of $F$ such that the pair of $D$ and $D^{\prime}$ is $\{\mathrm{T} 1, \mathrm{~T} 2\}$-dependent and the number of triple points of $D$ is equal to that of $D^{\prime}$.

The first author [12] observed the independence of the moves of types T1 and T2 as local moves, and showed that there is a pair of diagrams of the trivial $S^{2}-$ link with three (resp. four) components such that the pair is $\{\mathrm{T} 1\}$-dependent (resp. $\{\mathrm{T} 2\}$-dependent). Note that his proof does not work well for surface-links with two (resp. three) or less components. In Section 3, we will give the first example for the $\{\mathrm{T} 2\}$-dependence in the case of an $S^{2}-\mathrm{knot}$, and prove the following.

Theorem 1.5 There is an $S^{2}-k n o t$ with a pair of diagrams that is $\{\mathrm{T} 2\}$-dependent. In other words, any sequence of Roseman moves between the diagrams must involve at least one tetrahedral move.

Here are some questions for future research.

Problem 1.6 For each diagram $D$ of any surface-link $F$, is there a diagram $D^{\prime}$ of $F$ such that the pair $D$ and $D^{\prime}$ is $\{\mathrm{T} 2\}$-dependent?

Problem 1.7 Is there a pair of diagrams of a surface-link with two or less components that is $\{\mathrm{T} 1\}$-dependent? (See Remark 2.4.)

Problem 1.8 For each diagram $D$ of any surface-link $F$, is there a diagram $D^{\prime}$ of $F$ such that the pair $D$ and $D^{\prime}$ is $\{\mathrm{T} 1\}$-dependent?

Acknowledgments The authors would like to thank Michal Jabłonowski for referring us to Rosicki [18]. The second author is partially supported by Grant-in-Aid for Young Scientists (B) (No. 25800052), Japan Society for the Promotion of Science. The third author is partially supported by Grant-in-Aid for Scientific Research (C) (No. 26400082), Japan Society for the Promotion of Science.

\section{$2\{$ T1, T2 $\}$-dependent diagram pair}

In this section, we study $\{\mathrm{T} 1, \mathrm{~T} 2\}$-dependences of equivalent surface-link diagrams using the notion of coloring numbers, and prove Theorem 1.4. Throughout this paper, for a surface-link diagram $D$, let $\mathcal{S}_{D}$ denote the set of all sheets of $D$. Moreover, we represent the orientation of an oriented surface-link diagram by assigning its coorientation, depicted by an arrow which looks like the symbol $\Uparrow$ as in Figure 3, to each sheet of the diagram. 


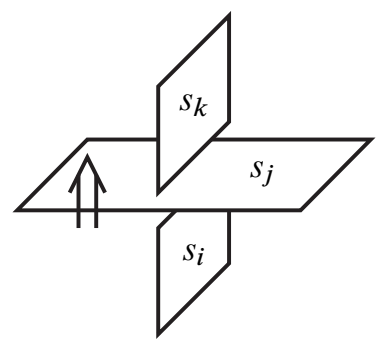

Figure 3: Coloring condition along a double point curve: $C\left(s_{i}\right) * C\left(s_{j}\right)=C\left(s_{k}\right)$

\section{A Coloring of surface-link diagrams}

Let $D$ be an oriented surface-link diagram and $\Omega$ a (nonempty) set with a binary operation $*: \Omega \times \Omega \rightarrow \Omega$. We say that a map $C: \mathcal{S}_{D} \rightarrow \Omega$ is an $\Omega$-coloring of $D$ if it satisfies the coloring condition $C\left(s_{i}\right) * C\left(s_{j}\right)=C\left(s_{k}\right)$ for each double point curve of $D$, where $s_{i}, s_{j}$ and $s_{k}$ are three sheets meeting at the double point curve such that the coorientation of $s_{j}$ points from $s_{i}$ to $s_{k}$ as in Figure 3. Note that there might be no $\Omega$-colorings of $D$ for given $D$ and $\Omega$. For an $\Omega$-coloring $C$, the image $C(s)$ of a sheet $s$ is called the color of $s$.

An $\Omega$-coloring is said to be trivial if it is a constant map. An oriented surface-link diagram has a trivial $\Omega$-coloring if and only if there exists an element $a \in \Omega$ such that $a * a=a$. Let $\operatorname{Col}_{\Omega}(D)$ denote the set of all $\Omega$-colorings of $D$. If $\Omega$ is finite, then we can count the number of the elements of $\operatorname{Col}_{\Omega}(D)$, and we call it the $\Omega$-coloring number of $D$ and denote it by $\# \operatorname{Col}_{\Omega}(D)$. Note that an $\Omega$-coloring number may depend on the choice of diagram for an oriented surface-link.

We say that $\Omega$ is involutory if for any $a, b \in \Omega,(a * b) * b=a$ holds. If $\Omega$ is involutory, the coloring condition does not depend on the coorientation of $s_{j}$, since $C\left(s_{i}\right) * C\left(s_{j}\right)=C\left(s_{k}\right)$ if and only if $C\left(s_{i}\right)=C\left(s_{k}\right) * C\left(s_{j}\right)$. Then an $\Omega$-coloring can be defined for an unoriented diagram of a (possibly nonorientable) surface-link.

\section{B Quandle and $\{$ T1, T2 $\}$-dependence}

The notion of quandles was introduced by Joyce [10] and Matveev [14] in 1982. We note that an involutory quandle, which was originally called a kei, had already been studied by Takasaki [15] in 1943. A quandle is a set $Q$ with a binary operation $*: Q \times Q \rightarrow Q$ satisfying the following three axioms:

(Q1) For any $a \in Q, a * a=a$.

(Q2) For any $a, b \in Q$, there exists a unique $c \in Q$ such that $c * b=a$.

(Q3) For any $a, b, c \in Q,(a * b) * c=(a * c) *(b * c)$. 
Axioms (Q1), (Q2) and (Q3) correspond to Reidemeister moves of types I, II and III respectively.

Example 2.1 Let $S_{4}$ denote the set $\{0,1,2,3\}$ with the binary operation defined by the following table:

\begin{tabular}{|c|c|c|c|c|}
\hline & & 0 & 1 & 2 \\
\hline & 0 & 0 & 2 & 3 \\
\hline$S_{4}$ & 1 & 3 & 1 & 0 \\
\hline & 2 & 1 & 3 & 2 \\
\hline & 3 & 2 & 0 & 1 \\
\hline
\end{tabular}

In this table, the $(i+1, j+1)$-entry represents $i * j$. Then $S_{4}$ satisfies the three axioms and we call it the tetrahedron quandle, which will be used in Section 3.

For a diagram $D$ of an oriented surface-link $F$, it is known that the $Q$-coloring number \# $\mathrm{Col}_{Q}(D)$ is an invariant of $F$ for a finite quandle $Q$; see, for example, [4; 18]. More precisely, it is known that

- the invariance under Roseman moves of types B1, B2 and BT comes from (Q1),

- the invariance under Roseman moves of types D1 and D2 comes from (Q2), and

- the invariance under Roseman moves of types T1 and T2 comes from (Q3).

Hence if we consider the case where a set $\Omega$ with a binary operation satisfies quandle axioms $(\mathrm{Q} 1)$ and $(\mathrm{Q} 2)$ but not $(\mathrm{Q} 3)$, then we have the following.

Lemma 2.2 Let $\Omega$ be a set with a binary operation satisfying quandle axioms (Q1) and $(\mathrm{Q} 2)$ but not $(\mathrm{Q} 3)$. If diagrams $D$ and $D^{\prime}$ of an oriented surface-link are not $\{\mathrm{T} 1, \mathrm{~T} 2\}$-dependent, then $\# \mathrm{Col}_{\Omega}(D)=\# \mathrm{Col}_{\Omega}\left(D^{\prime}\right)$ holds.

Proof Since $D$ and $D^{\prime}$ are not $\{\mathrm{T} 1, \mathrm{~T} 2\}$-dependent, there exists a finite sequence of Roseman moves without T1 - and T2-moves. The quandle axiom (Q3) affects only the invariance by $\mathrm{T} 1-$ and $\mathrm{T} 2-$ moves, and hence $\# \operatorname{Col}_{\Omega}(D)=\# \operatorname{Col}_{\Omega}\left(D^{\prime}\right)$ holds.

\section{C Proof of Theorem 1.4}

Let $X$ be the set $\{0,1,2\}$ with the binary operation $*: X \times X \rightarrow X$ defined by the following table:

\begin{tabular}{l|lll} 
& 0 & 1 & 2 \\
\hline 0 & 0 & 2 & 1 \\
1 & 1 & 1 & 0 \\
2 & 2 & 0 & 2
\end{tabular}



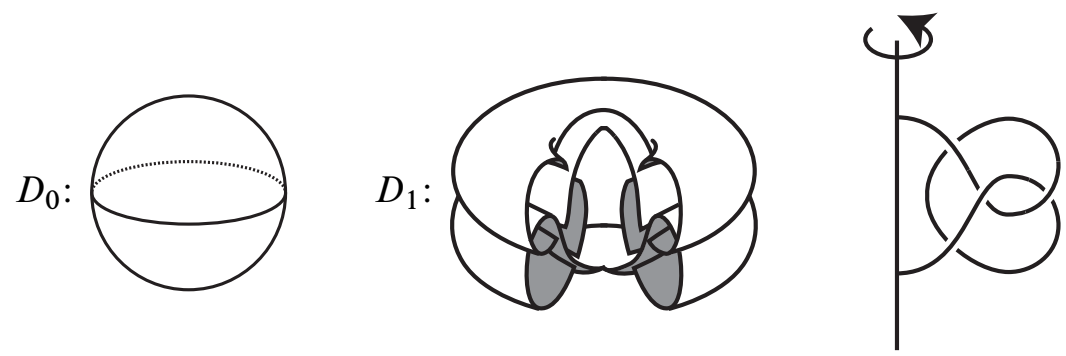

Figure 4: Two diagrams $D_{0}$ and $D_{1}$ of the trivial $S^{2}-$ knot

In this table, the $(i+1, j+1)$-entry represents $i * j$. The binary operation of $X$ satisfies the quandle axioms $(\mathrm{Q} 1)$ and $(\mathrm{Q} 2)$ but not $(\mathrm{Q} 3)$. For example, $(0 * 1) * 2=2 * 2=2$ and $(0 * 2) *(1 * 2)=1 * 0=1$. Since the binary operation of $X$ is involutory, we can apply Lemma 2.2 to unoriented diagrams of a (possibly nonorientable) surface-link. We found that the same binary operation was used by Cheng and Gao in [5] for the study of classical knot diagrams. They used $X$-coloring numbers to investigate the independence of Reidemeister move of type III.

Proposition 2.3 Let $D_{0}$ be the trivial diagram of the trivial $S^{2}-k n o t$, that is, $D_{0}$ is the standard 2-sphere in $\mathbb{R}^{3}$. There exists a diagram $D_{1}$ of the trivial $S^{2}-k n o t$ such that the pair of $D_{0}$ and $D_{1}$ is $\{\mathrm{T} 1, \mathrm{~T} 2\}$-dependent, and they have no triple points.

Proof Let $D_{1}$ be the $S^{2}$-knot diagram obtained by spinning the tangle diagram in Figure 4. Since the tangle diagram in Figure 4 represents the unknotted tangle, $D_{1}$ represents the trivial $S^{2}$-knot. Moreover, $D_{0}$ and $D_{1}$ have no triple points. Since $D_{0}$ consists of a single sheet and $X$ consists of three elements, $D_{0}$ has three trivial $X$-colorings, and hence we have $\mathbb{C}_{\operatorname{Col}_{X}}\left(D_{0}\right)=3$. On the other hand, $D_{1}$ has three trivial $X$-colorings and four nontrivial ones, and hence we have $\# \operatorname{Col}_{X}\left(D_{1}\right)=7$. Thus, we have $\# \operatorname{Col}_{X}\left(D_{0}\right) \neq \# \operatorname{Col}_{X}\left(D_{1}\right)$, which implies that the pair of $D_{0}$ and $D_{1}$ is $\{\mathrm{T} 1, \mathrm{~T} 2\}$-dependent by Lemma 2.2 .

Remark 2.4 Since there exists a finite sequence of Roseman moves of types B1, B2 and T1 between $D_{0}$ and $D_{1}$ above, the pair of them is not $\{\mathrm{T} 2\}$-dependent. It is therefore natural to ask the following question: is the pair of $D_{0}$ and $D_{1}$ above $\{\mathrm{T} 1\}$-dependent? (See Problem 1.7.)

Proof of Theorem 1.4 For any diagram $D$ of a surface-link $F$, we set a diagram $D^{\prime}$ to be a connected sum $D \sharp D_{1}$ of $D$ and $D_{1}$ as in Figure 5, where $D_{1}$ is the diagram of the trivial $S^{2}$-knot in Figure 4. Then $D^{\prime}$ is a diagram of $F$ and the number of triple points of $D$ is equal to that of $D^{\prime}$. Let $s_{1}$ be the sheet of $D_{1}$ where we have performed the 


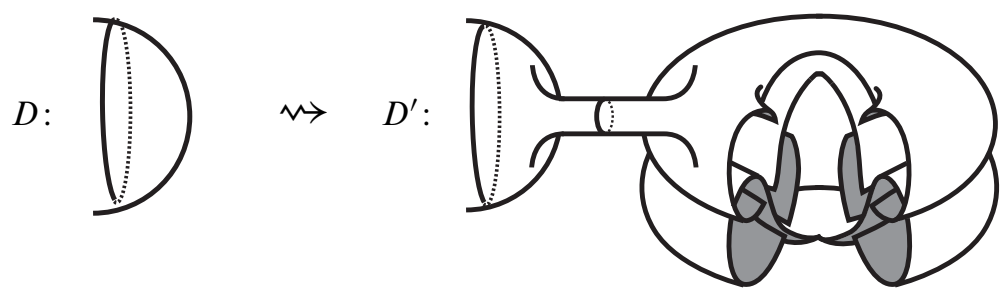

Figure 5: The diagrams $D$ and $D^{\prime}=D \sharp D_{1}$ of the surface-link $F$

connected sum. Then for each element, say $a$, in $X$, there are at least two $X$-colorings of $D_{1}$ such that the sheet $s_{1}$ receives the color $a$. Hence the $X$-coloring number of $D^{\prime}$ is at least twice of that of $D$, that is, $\# \operatorname{Col}_{X}\left(D^{\prime}\right) \geq 2 \times \# \operatorname{Col}_{X}(D)>\# \operatorname{Col}_{X}(D) \geq 3$. By Lemma 2.2, we conclude that the pair of $D$ and $D^{\prime}$ is $\{\mathrm{T} 1, \mathrm{~T} 2\}$-dependent.

Remark 2.5 Theorem 1.4 is a generalization of Jabłonowski's result [9, Theorem 1.3]. However we cannot prove that the pair of his surface-link diagrams is $\{\mathrm{T} 1, \mathrm{~T} 2\}-$ dependent by using $X$-coloring number.

\section{3 \{T2\}-dependent diagram pair}

Carter et al [1] studied the number of Reidemeister moves of type III needed to move between two diagrams of the same classical knot using a modification of quandle cocycle invariants. In this section, we study the $\{\mathrm{T} 2\}$-dependence of equivalent $S^{2}-$ knot diagrams using a similar idea to the one in [1].

\section{A Multiset $\Phi_{\theta}(D)$}

Let $D$ be a diagram of an oriented surface-link and $\tau$ a triple point of $D$. For a small neighborhood of $\tau$, the complement of $D$ is divided into eight regions. We denote by $R$ one of the eight regions from which all coorientations of the three sheets point outward. Let $s_{T}, s_{M}$ and $s_{B}$ be the top, middle and bottom sheets of $\tau$ respectively, which bounds the region $R$. Note that when three sheets form a triple point, they have positions top, middle and bottom with respect to the height information of the $4^{\text {th }}$ coordinate dropped by the projection from $\mathbb{R}^{4}$ to $\mathbb{R}^{3}$.

Let $Q$ be a quandle and $A$ an abelian group. We set a function $\theta: Q^{3} \rightarrow A$. For a $Q$-coloring $C: \mathcal{S}_{D} \rightarrow Q$ of the diagram $D$, the (Boltzmann) weight $\mathrm{B}_{\theta}(\tau, C)$ of $\tau$ is defined to be $\varepsilon \theta\left(C\left(s_{B}\right), C\left(s_{M}\right), C\left(s_{T}\right)\right) \in A$, where $\varepsilon=+1$ if the coorientations of $s_{T}, s_{M}$ and $s_{B}$ in this order matches the orientation of $\mathbb{R}^{3}$ and $\varepsilon=-1$ otherwise. 
We denote by $W_{\theta}(D, C)$ the sum of the weights of all triple points of $D$, that is,

$$
W_{\theta}(D, C)=\sum_{\tau \in \text { \{triple points of } D\}} \mathrm{B}_{\theta}(\tau, C) \in A .
$$

Let $D$ and $D^{\prime}$ be oriented surface-link diagrams such that $D^{\prime}$ is obtained from $D$ by a single T2-move. For a $Q$-coloring $C$ of $D$, there is a unique $Q$-coloring $C^{\prime}$ of $D^{\prime}$ such that these two $Q$-colorings coincide in the complement of the 3-ball where the T2-move is performed. For such pairs of $(D, C)$ and $\left(D^{\prime}, C^{\prime}\right)$, we have the following.

Lemma 3.1 [2] There exist some $a, b, c, d \in Q$ such that

$$
\begin{aligned}
W_{\theta}(D, C)-W_{\theta}\left(D^{\prime}, C^{\prime}\right)= & \pm[\theta(a, c, d)-\theta(a * b, c, d)-\theta(a, b, d) \\
& +\theta(a * c, b * c, d)+\theta(a, b, c)-\theta(a * d, b * d, c * d)]
\end{aligned}
$$

for the pairs of $Q$-colored diagrams $(D, C)$ and $\left(D^{\prime}, C^{\prime}\right)$ related by a single T2-move.

We denote by $\Phi_{\theta}(D)$ the multiset $\left\{W_{\theta}(D, C) \mid C \in \mathrm{Col}_{Q}(D)\right\}$. For an unoriented diagram $D$ of an orientable surface-link, by considering all possible orientations of $D$, we define the multiset (of multisets) $\Phi_{\theta}^{\text {unori }}(D)$ by

$$
\left\{\Phi_{\theta}(\vec{D}) \mid \vec{D} \in\{\text { oriented diagrams representing } D\}\right\} \text {. }
$$

\section{B Quandle 3-cocycle condition and \{T2\}-dependence}

For a quandle $Q$ and an abelian group $A$, a function $\theta: Q^{3} \rightarrow A$ is called a quandle 3 -cocycle if it satisfies the following conditions:

(i) For any $a, b \in Q, \theta(a, a, b)=0$ and $\theta(a, b, b)=0$.

(ii) For any $a, b, c, d \in Q$,

$\theta(a, c, d)-\theta(a * b, c, d)-\theta(a, b, d)+\theta(a * c, b * c, d)+\theta(a, b, c)-\theta(a * d, b * d, c * d)=0$.

These conditions are called the quandle 3-cocycle conditions, which arise from quandle cohomology theories [2].

Lemma 3.1 shows that condition (ii) coincides with the difference of the sum of the weights for two $Q$-colored surface-link diagrams related by a single T2-move. This implies that when a function $\theta: Q^{3} \rightarrow A$ satisfies condition (ii), the multiset $\Phi_{\theta}(D)$ for a diagram $D$ of an oriented surface-link is unchanged under T2-moves. Additionally, we can easily check that condition (ii) does not affect the other types of Roseman moves. We can similarly see that condition (i) guarantees the invariance of $\Phi_{\theta}(D)$ 
under BT-moves. It is shown in [2] that if the function $\theta$ satisfies the quandle 3cocycle conditions, the multiset $\Phi_{\theta}(D)$ is independent of the choice of diagram, and thus, it is an invariant of oriented surface-links. Now, we consider the case where a function $\theta: Q^{3} \rightarrow A$ satisfies condition (i) but not (ii).

Proposition 3.2 For a quandle $Q$ and an abelian group $A$, we set a function $\theta: Q^{3} \rightarrow A$ satisfying quandle 3-cocycle condition (i) but not (ii). Let $D$ and $D^{\prime}$ be oriented diagrams which represent the same oriented surface-link. If $D$ and $D^{\prime}$ are not $\{\mathrm{T} 2\}-$ dependent, then $\Phi_{\theta}(D)=\Phi_{\theta}\left(D^{\prime}\right)$.

Proof Since $D$ and $D^{\prime}$ are not $\{\mathrm{T} 2\}$-dependent, there exists a finite sequence of Roseman moves without T2-moves. The quandle 3-cocycle condition (ii) affects only the invariance by T2-moves, and hence, $\Phi_{\theta}(D)=\Phi_{\theta}\left(D^{\prime}\right)$ holds.

For an unoriented diagram of an orientable surface-link, we have the following.

Corollary 3.3 For a quandle $Q$ and an abelian group $A$, we set a function $\theta: Q^{3} \rightarrow A$ satisfying quandle 3-cocycle condition (i) but not (ii). Let $D$ and $D^{\prime}$ be unoriented diagrams which represent the same orientable surface-link. If $D$ and $D^{\prime}$ are not $\{\mathrm{T} 2\}$-dependent, then $\Phi_{\theta}^{\text {unori }}(D)=\Phi_{\theta}^{\text {unori }}\left(D^{\prime}\right)$.

\section{C Proof of Theorem 1.5}

To prove Theorem 1.5, we will construct two concrete $S^{2}$-knot diagrams using the deform-spinning method defined by Litherland [13], which is reviewed below. This is a method of constructing $S^{2}$-knots from a classical knot such that a deformation can be applied during the spinning process. Note that the twist-spinning method by Zeeman [24] and the roll-spinning method by Fox [6] are special cases of the deform-spinning method.

For a classical knot $K$, consider a properly embedded arc $K_{0}$ in the unit 3-ball $B^{3}$ such that $K$ is obtained from $K_{0}$ by connecting the boundary points by a simple arc in $\partial B^{3}$. Let $f_{t}: B^{3} \rightarrow B^{3}(t \in[0,1])$ be an isotopy of $B^{3}$ rel $\partial B^{3}$ such that $f_{1}\left(K_{0}\right)=K_{0}$. Define

$$
\left(S^{4}, F\right)=\bigcup_{t \in[0,1]}\left(B^{3}, f_{t}\left(K_{0}\right)\right) / \sim,
$$

where $\sim$ stands for

$$
\begin{cases}\left(f_{0}(\boldsymbol{x}), 0\right) \sim\left(f_{1}(\boldsymbol{x}), 1\right) & \text { for } \boldsymbol{x} \in B^{3}, \\ (\boldsymbol{x}, t) \sim\left(\boldsymbol{x}, t^{\prime}\right) & \text { for } \boldsymbol{x} \in \partial B^{3} \text { and } t, t^{\prime} \in[0,1] .\end{cases}
$$




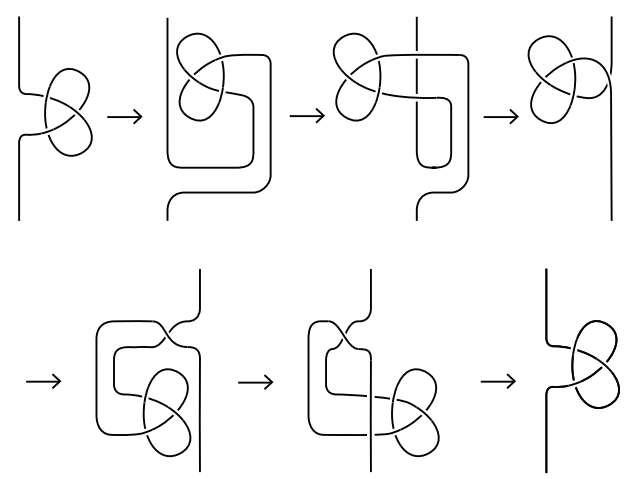

Figure 6: The motion picture of the 1-twist of $\tau^{3}\left(3_{1}\right)$

Then $F$ is a $2-$ sphere embedded in a 4 -sphere $S^{4}$. Removing a point from $S^{4} \backslash F$, we have an $S^{2}$-knot $F$ in $\mathbb{R}^{4}$. We call $F$ a deform-spun $S^{2}-k n o t$ of $K$. Note that the family $\left\{f_{t}\left(K_{0}\right)\right\}_{t \in[0,1]}$ is a motion picture of tangles in $B^{3}$ which describes $F$, and thus, any deform-spun $S^{2}$-knot can be described by a motion picture of tangles in $B^{3}$.

Let $F$ be a deform-spun $S^{2}$-knot. Let $p: \mathbb{R}^{4} \rightarrow \mathbb{R}^{3}$ be the projection induced by the natural projection, say also $p$, from $B^{3}$ to $B^{2}$ dropping the $3^{\text {rd }}$ coordinate. For each $t \in[0,1]$, the image $p\left(f_{t}\left(K_{0}\right)\right)$ equipped with the height information is a tangle diagram in $B^{2}$, and the family $\left\{p\left(f_{t}\left(K_{0}\right)\right)\right\}_{t \in[0,1]}$ with the height information is a motion picture of tangle diagrams in $B^{2}$ which describes a diagram of $F$. Thus we can obtain a diagram of $F$ by a motion picture of tangle diagrams in $B^{2}$. Note that each Reidemeister move of type III in a motion picture produces a triple point of the corresponding generic projection. Furthermore, for a quandle $Q$, we can also see a $Q-$ colored diagram of $F$ by taking a motion picture of $Q$-colored tangle diagrams in $B^{2}$.

Let $\tau^{3}\left(3_{1}\right)$ denote the deform-spun $S^{2}$-knot described by the threefold repetition of the motion of tangle diagrams in Figure 6, where the tangle diagrams in Figure 6 represent the left-handed trefoil knot $3_{1}$. We note that $\tau^{3}\left(3_{1}\right)$ is usually called the 3 -twist-spun $S^{2}-k n o t$ of $3_{1}$ and that this method of drawing motion pictures for twist-spun $S^{2}-$ knots was first given in [20]. Let $\rho^{1 / 2}\left(4_{1}\right)$ be the deform-spun $S^{2}-$ knot described by the motion picture of tangle diagrams in Figure 7, where the tangle diagrams in Figure 7 represent the figure-eight knot $4_{1}$. We note that $\rho^{1 / 2}\left(4_{1}\right)$ is usually called the (half-) roll-spun $S^{2}-$ knot of $4_{1}$.

Proof of Theorem 1.5 Let $D$ denote the diagram of $\tau^{3}\left(3_{1}\right)$ described by the threefold repetition of the motion of tangle diagrams in Figure 6, and let $D^{\prime}$ be the diagram of $\rho^{1 / 2}\left(4_{1}\right)$ described by the motion picture of tangle diagrams in Figure 7 . We note that $D$ has eighteen triple points and that $D^{\prime}$ has twelve triple points. Since it is shown in 


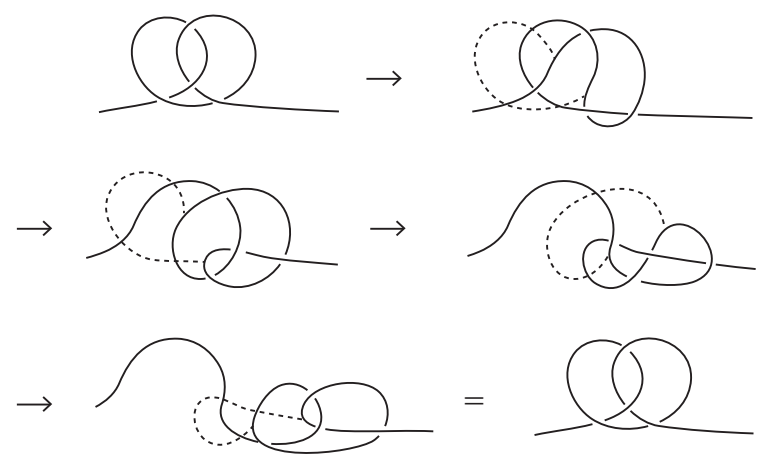

Figure 7: The motion picture of $\rho^{1 / 2}\left(4_{1}\right)$

[11; 22] that two $S^{2}-$ knots $\tau^{3}\left(3_{1}\right)$ and $\rho^{1 / 2}\left(4_{1}\right)$ are equivalent, $D$ and $D^{\prime}$ are also equivalent as $S^{2}$-knot diagrams. In what follows, we focus on proving that the pair of $D$ and $D^{\prime}$ is $\{\mathrm{T} 2\}$-dependent.

Let $S_{4}$ be the tetrahedron quandle given in Example 2.1 and define a map $\theta:\left(S_{4}\right)^{3} \rightarrow \mathbb{Z}$ by $\theta(x, y, z)=(x-y)(y-z)$. Note that the map $\theta$ satisfies the quandle 3-cocycle condition (i) but not (ii). To prove this theorem, it is enough to show that the multiset $\Phi_{\theta}^{\text {unori }}(D)$ does not coincide with the multiset $\Phi_{\theta}^{\text {unori }}\left(D^{\prime}\right)$ by Corollary 3.3. More precisely, for some oriented diagram $\vec{D}^{\prime}$ of $D^{\prime}$, we show that there exists an $S_{4}-$ coloring $C^{\prime}$ of $\vec{D}^{\prime}$ such that the weight sum $W_{\theta}\left(\vec{D}^{\prime}, C^{\prime}\right)$ is nonzero. On the other hand, for any oriented diagram $\vec{D}$ of $D$ and any $S_{4}$-coloring $C$ of $\vec{D}$, we show that the weight sum $W_{\theta}(\vec{D}, C)$ is zero, that is, $\Phi_{\theta}^{\text {unori }}(D)=\left\{0_{16}, 0_{16}\right\}$, where $0_{16}$ is the multiset composed of 16 zeros.

First, consider the multiset $\Phi_{\theta}^{\text {unori }}(D)$. (We refer to [20] for computation of quandle cocycle invariants of twist-spun $S^{2}$-knots.) Set the orientation of $D$ as shown in Figure 8 and we denote by $\vec{D}$ the oriented diagram. Note that Figure 8 shows the first 1 -twist of $\vec{D}$. We also note that each of the deformations (M1) and (M2) produces three triple points. For any elements $a, b \in S_{4}$, Figure 8 represents an $S_{4}$-coloring (of the first 1 -twist) of $\vec{D}$, that is, the assignment of elements of $S_{4}$ satisfies the $S_{4}$-coloring condition. Note that when we replace each element, say $x \in S_{4}$, appearing in Figure 8 by $x * a$ (resp. $(x * a) * a)$, the motion of the replaced $S_{4}$-colored tangle diagrams describes the $S_{4}$-coloring of the second (resp. third) 1 -twist of $\vec{D}$. We also note that the arc colored by $a * b$ just before the deformation (M2) in Figure 8 receives the color $b$ just after the same deformation, because $(a * b) * a=b$ holds for any $a, b \in S_{4}$. Since $((x * a) * a) * a=b$ holds for any $x, a \in S_{4}$, the last $S_{4}$-colored tangle diagram of the third 1-twist of $\vec{D}$ coincides with the first one in Figure 8 . 


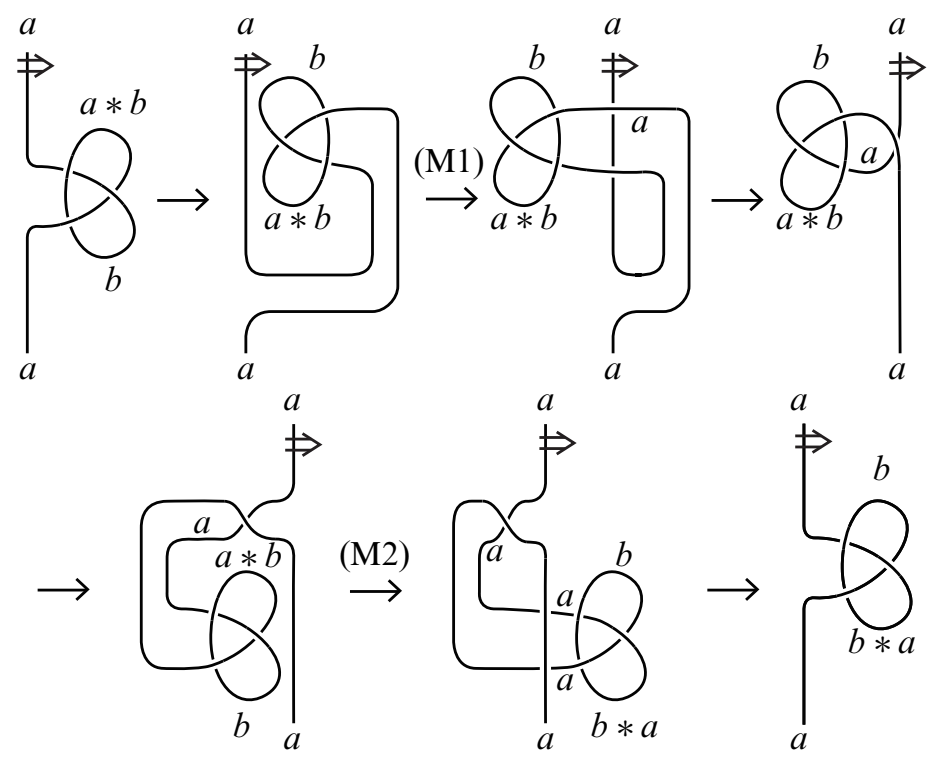

Figure 8: $S_{4}$-coloring of the 1 -twist of $\tau^{3}\left(3_{1}\right)$

For the first 1-twist, the sum of the weights of the six triple points appearing in this step is

$$
\begin{aligned}
-\theta(b, b, a * b) & -\theta(b, a, b)-\theta(b, a * b, a) \\
& +\theta(b, a * b, a)+\theta(a, b, a)+\theta(a * b, a, a),
\end{aligned}
$$

where the first row is obtained from the deformation (M1) in Figure 8 and the second row from (M2). It is easy to see that the third term and the fourth term cancel and the first term and the last term are zero. Since

$$
-\theta(b, a, b)+\theta(a, b, a)=-(b-a)(a-b)+(a-b)(b-a)=0,
$$

the formula (1) is equal to 0 . Similarly, for the second 1-twist, we also have

$$
\begin{aligned}
-\theta(b * a, b * a, b) & -\theta(b * a, a, b * a)-\theta(b * a, b, a) \\
& +\theta(b * a, b, a)+\theta(a, b * a, a)+\theta(b, a, a)=0,
\end{aligned}
$$

and for the third 1-twist, we also have

$$
\begin{aligned}
-\theta(a * b, a * b, b * a) & -\theta(a * b, a, a * b)-\theta(a * b, b * a, a) \\
& +\theta(a * b, b * a, a)+\theta(a, a * b, a)+\theta(b * a, a, a)=0 .
\end{aligned}
$$

Therefore the sum of the weight of all eighteen triple points of $\vec{D}$ is zero. This implies that $\Phi_{\theta}(\vec{D})=\left\{0_{16}\right\}$. In the case where we set the reversed orientation for $D$, we can similarly see that the multiset is $\left\{0_{16}\right\}$. Hence, we have $\Phi_{\theta}^{\text {unori }}(D)=\left\{0_{16}, 0_{16}\right\}$. 


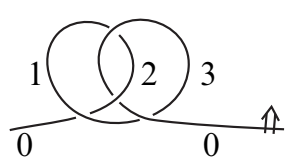

$\stackrel{(\mathrm{M} 1)}{\longrightarrow}$

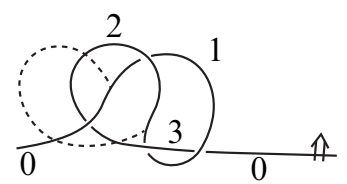

$\stackrel{(\mathrm{M} 2)}{\longrightarrow}$

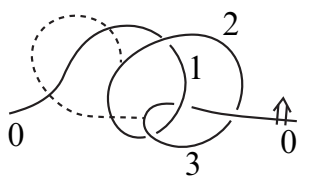

$\stackrel{(\mathrm{M} 3)}{\longrightarrow}$

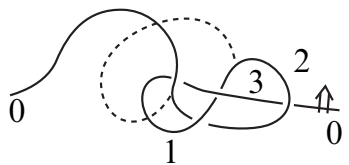

(M4)
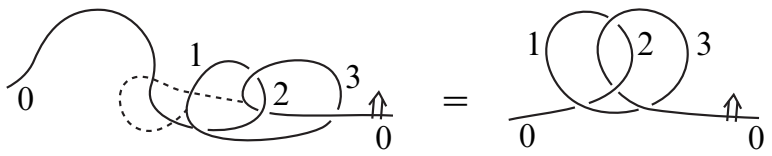

Figure 9: $S_{4}$-coloring of $\rho^{1 / 2}\left(4_{1}\right)$

Let us consider the multiset $\Phi_{\theta}^{\text {unori }}\left(D^{\prime}\right)$. (We refer to [8] for computation of quandle cocycle invariants of roll-spun $S^{2}$-knots.) Set the orientation and the $S_{4}$-coloring of $D^{\prime}$ as shown in Figure 9. We denote by $\vec{D}^{\prime}$ the oriented diagram of $D^{\prime}$. Note that each of the deformations (M1)-(M4) produces three triple points. By direct calculation, we can see that the sum of the weights is

$$
\begin{aligned}
& \theta(3,0,1)+\theta(1,2,1)-\theta(1,3,1) \\
+ & \theta(0,1,2)-\theta(2,1,3)-\theta(2,3,1) \\
+ & \theta(3,1,2)+\theta(1,3,2)-\theta(3,0,2) \\
+ & \theta(1,0,1)-\theta(3,1,3)-\theta(1,0,2)=12,
\end{aligned}
$$

where the first, second, third and last rows of the left-hand side are obtained from the deformations (M1), (M2), (M3) and (M4) in Figure 9, respectively. This implies that $\Phi_{\theta}\left(\vec{D}^{\prime}\right)$ has a nonzero element, and we have $\Phi_{\theta}^{\text {unori }}\left(D^{\prime}\right) \neq \Phi_{\theta}^{\text {unori }}(D)=\left\{0_{16}, 0_{16}\right\}$.

By taking the connected sum of $D$ and $D^{\prime}$ above with the trivial orientable surface-knot diagram, we can show the following in a similar way.

Corollary 3.4 For any positive integer $g$, there is an orientable surface-knot of genus $g$ with a pair of diagrams that is $\{\mathrm{T} 2\}$-dependent. In other words, any sequence of Roseman moves between them must involve at least one tetrahedral move.

\section{References}

[1] J S Carter, M Elhamdadi, M Saito, S Satoh, A lower bound for the number of Reidemeister moves of type III, Topology Appl. 153 (2006) 2788-2794 MR2248382 
[2] J S Carter, D Jelsovsky, S Kamada, L Langford, M Saito, Quandle cohomology and state-sum invariants of knotted curves and surfaces, Trans. Amer. Math. Soc. 355 (2003) 3947-3989 MR1990571

[3] J S Carter, M Saito, Knotted surfaces and their diagrams, Mathematical Surveys and Monographs 55, Amer. Math. Soc. (1998) MR1487374

[4] S Carter, S Kamada, M Saito, Surfaces in 4-space, Encyclopaedia of Mathematical Sciences 142, Springer, Berlin (2004) MR2060067

[5] Z Cheng, H Gao, A note on the independence of Reidemeister moves, J. Knot Theory Ramifications 21 (2012) 1220001, 7 MR2926565

[6] R H Fox, Rolling, Bull. Amer. Math. Soc. 72 (1966) 162-164 MR0184221

[7] T Homma, T Nagase, On elementary deformations of maps of surfaces into 3manifolds, I, Yokohama Math. J. 33 (1985) 103-119 MR817976

[8] M Iwakiri, S Satoh, Quandle cocycle invariants of roll-spun knots, preprint 1776, RIMS (2011) Available at http://www.kurims.kyoto-u.ac.jp/ kyodo/ kokyuroku/contents/pdf/1766-04.pdf

[9] M Jabłonowski, Knotted surfaces and equivalencies of their diagrams without triple points, J. Knot Theory Ramifications 21 (2012) 1250019, 6 MR2887654

[10] D Joyce, A classifying invariant of knots, the knot quandle, J. Pure Appl. Algebra 23 (1982) 37-65 MR638121

[11] T Kanenobu, Untwisted deform-spun knots: examples of symmetry-spun 2-knots, from: "Transformation groups", (K Kawakubo, editor), Lecture Notes in Math. 1375, Springer, Berlin (1989) 145-167 MR1006689

[12] K Kawamura, On relationship between seven types of Roseman moves, Topology Appl. 196 (2015) 551-557 MR3430997

[13] R A Litherland, Deforming twist-spun knots, Trans. Amer. Math. Soc. 250 (1979) 311-331 MR530058

[14] S V Matveev, Distributive groupoids in knot theory, Mat. Sb. 119(161) (1982) 78-88, 160 MR672410 In Russian; translated in USSR-Sb. 47 (1984) no. 1, 73-83

[15] T Mituhisa, Abstraction of symmetric transformations, Tôhoku Math. J. 49 (1943) 145-207 MR0021002

[16] K Oshiro, K Tanaka, On rack colorings for surface-knot diagrams without branch points, Topology Appl. 196 (2015) 921-930 MR3431027

[17] D Roseman, Reidemeister-type moves for surfaces in four-dimensional space, from: "Knot theory", (VFR Jones, J Kania-Bartoszyńska, J H Przytycki, V G Traczyk, Pawełand Turaev, editors), Banach Center Publ. 42, Polish Acad. Sci., Warsaw (1998) 347-380 MR1634466 
[18] W Rosicki, Some simple invariants of the position of a surface in $\mathbf{R}^{4}$, Bull. Polish Acad. Sci. Math. 46 (1998) 335-344 MR1657152

[19] S Satoh, Double decker sets of generic surfaces in 3-space as homology classes, Illinois J. Math. 45 (2001) 823-832 MR1879237

[20] S Satoh, Surface diagrams of twist-spun 2-knots, J. Knot Theory Ramifications 11 (2002) 413-430 MR1905695 Knots 2000 Korea, Vol. 1 (Yongpyong)

[21] M Takase, K Tanaka, Regular-equivalence of 2-knot diagrams and sphere eversions, Math. Proc. Cambridge Philos. Soc. 161 (2016) 237-246 MR3530506

[22] M Teragaito, Twisting and rolling, from: "Problems and recent results in lowdimensional topology", RIMS Kokyuroku 636, Research Institute for Mathematical Sciences, Kyoto University (1987) 153-169

[23] T Yashiro, A note on Roseman moves, Kobe J. Math. 22 (2005) 31-38 MR2202688

[24] E C Zeeman, Twisting spun knots, Trans. Amer. Math. Soc. 115 (1965) 471-495 MR0195085

Department of Mathematics, Osaka City University

3-3-138 Sugimoto-cho, Sumiyoshi-ku, Osaka 558-8585, Japan

Department of Information and Communication Sciences, Sophia University

7-1 Kioi-cho, Chiyoda-ku, Tokyo 102-8554, Japan

Department of Mathematics, Tokyo Gakugei University

4-1-1 Nukuikita-machi, Koganei-shi, Tokyo 184-8501, Japan

k.kawamura0403@gmail.com, oshirok@sophia.ac.jp, kotanaka@u-gakugei.ac.jp

Received: 17 November 2015 Revised: 28 December 2015 